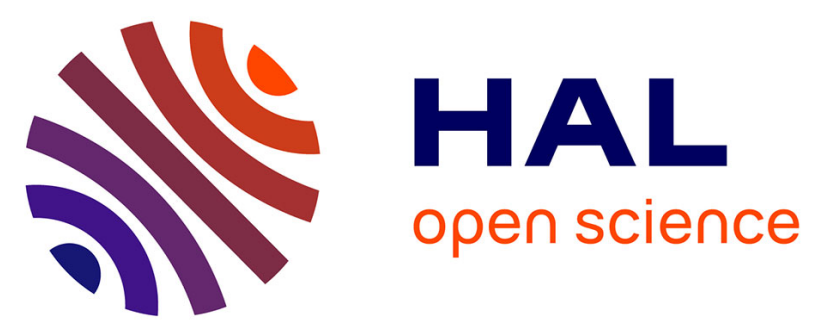

\title{
Vasoactive intestinal peptide is a local mediator in a gut-brain neural axis activating intestinal gluconeogenesis
}

Filipe de Vadder, Flora Plessier, Amandine Gautier-Stein, Gilles Mithieux

\section{- To cite this version:}

Filipe de Vadder, Flora Plessier, Amandine Gautier-Stein, Gilles Mithieux. Vasoactive intestinal peptide is a local mediator in a gut-brain neural axis activating intestinal gluconeogenesis. Neurogastroenterology \& Motility, 2015, 27 (3), pp.443-448. 10.1016/j.molmet.2014.12.009 . inserm-01350922

\section{HAL Id: inserm-01350922 https://www.hal.inserm.fr/inserm-01350922}

Submitted on 2 Aug 2016

HAL is a multi-disciplinary open access archive for the deposit and dissemination of scientific research documents, whether they are published or not. The documents may come from teaching and research institutions in France or abroad, or from public or private research centers.
L'archive ouverte pluridisciplinaire HAL, est destinée au dépôt et à la diffusion de documents scientifiques de niveau recherche, publiés ou non, émanant des établissements d'enseignement et de recherche français ou étrangers, des laboratoires publics ou privés. 


\title{
VASOACTIVE INTESTINAL PEPTIDE IS A LOCAL MEDIATOR IN A GUT- BRAIN NEURAL AXIS ACTIVATING INTESTINAL GLUCONEOGENESIS
}

Filipe De Vadder ${ }^{1,2,3}$, Flora Plessier ${ }^{1,2,3}$, Amandine Gautier-Stein ${ }^{1,2,3}$, Gilles Mithieux ${ }^{1,2,3}$

${ }^{1}$ Institut de la Santé et de la Recherche Médicale, U855, Lyon, 69372, France

${ }^{2}$ Université de Lyon, Lyon, 69008, France

${ }^{3}$ Université Lyon 1, Villeurbanne, 69622, France

Correspondence to Gilles Mithieux, UMR INSERM 855, Faculté de Médecine Laennec Lyon-Est, 7-11 rue Paradin, 69372 Lyon Cedex 08, France.

Tel: +334787787 88. Fax: +33478778762

gilles.mithieux@inserm.fr

\begin{abstract}
Intestinal gluconeogenesis (IGN) promotes metabolic benefits through activation of a gutbrain neural axis. However, the local mediator activating gluconeogenic genes in the enterocytes remains unknown. We show that (1) VIP signaling through VPAC1 receptor activates the intestinal glucose-6-phosphatase gene in vivo, (2) the activation of IGN by propionate is counteracted by VPAC1 antagonism, and (3) VIP-positive intrinsic neurons in the submucosal plexus are increased under the action of propionate. These data support the role of VIP as a local neuromodulator released by intrinsic enteric neurons and responsible for the induction through a VPAC1 receptor-dependent mechanism in enterocytes.
\end{abstract}

\section{KEYWORDS}

Intestinal gluconeogenesis; vasoactive intestinal peptide; gut-brain axis; glucose-6phosphatase

\section{KEY MESSAGES}


- Vasoactive intestinal peptide is a local neuromodulator released by enteric neurons in response to propionate feeding. This in turn activates intestinal gluconeogenesis, a key process in the regulation of glucose and energy balance.

- Aims/goals: We sought to determine the neural mechanisms by which intestinal glucose production is activated following a propionate-enriched diet.

- We fed rats standard or propionate-enriched diet for two weeks and we evaluated the activation of intestinal gluconeogenesis genes in response to these diets and to VIP signaling (i.e. exogenous VIP and VPAC1 receptor antagonist). Furthermore, we quantified VIP-positive neurons in the enteric nervous system in response to propionate feeding.

- When injected with VIP, rats fed a control diet show an activation of intestinal gluconeogenesis genes. This activation was also present in propionate-fed rats but was reversed by VPAC1 receptor antagonist injection. VIP enteric submucosal neurons were also more numerous in propionate-fed rats. 


\section{INTRODUCTION}

The gut-brain axis plays a critical role in the regulation of metabolic homeostasis. Intestinal gluconeogenesis (IGN) is a newly described process that promotes a decrease of hunger and an improvement of insulin sensitivity, while decreasing hepatic glucose production(1). The IGN function is described in humans, especially after gastric bypass surgery or during the an-hepatic phase of liver transplantation(2). Intestinal gluconeogenesis is activated when feeding protein $(3,4)$ - or dietary fiber(5)-enriched diets. Specifically, in the case of dietary fiber, microbial-generated propionate binds to free fatty acid receptor 3 (FFAR3) in the nerve endings of the portal vein wall. This leads to the activation of a gutbrain reflex arc, inducing in the end the expression of gluconeogenic genes (glucose-6phosphatase, G6Pase; phosphoenolpyruate carboxykinase, PEPCK)(5). However, the local enteric relay responsible for the activation of IGN genes in the enterocytes remains to be elucidated. We hypothesized that the release of a local gut neurotransmitter by enteric nervous plexuses in response to a signal from the central nervous system could be involved.

Vasoactive intestinal peptide (VIP) is a small peptide that acts as a neurotransmitter and paracrine neurohormone in non-adrenergic neurons of the enteric nervous system(6). VIP neurons innervate the gut epithelium, and VIP specifically increases cAMP levels in enterocytes through binding to $\mathrm{G}_{\mathrm{s}}$-coupled VPAC1 receptor(7). In vitro studies in Caco-2 cells overexpressing the catalytic subunit of G6Pase (G6PC) have shown that transcription of $G 6 P C$ is activated by cAMP-mediated signaling in the intestine(8). Interestingly, isolated intestinal epithelial cells from rat exhibit a high adenylate cyclase sensitivity to VIP(9), and culturing Caco-2 cells overexpressing G6PC in the presence of VIP up-regulates the transcription of the gene(8). These data suggest that local release of VIP by enteric neurons may be involved in activation of IGN genes in the gut epithelium.

To test this hypothesis, we fed rats for two weeks with either standard chow or propionate-enriched chow to induce IGN gene expression (see Methods). We investigated the in vivo implications of VIP on IGN gene expression and cAMP-mediated signaling in the intestine.

\section{RESULTS AND DISCUSSION}

Rats that were fed on standard chow and received a VIP injection had about 2.5-fold higher G6Pase activity in the jejunum compared with rats that received a saline injection. In 
line with previous data(5), propionate feeding strongly induced G6Pase activity. It is noteworthy that an injection of VPAC1 antagonist PG 97-269 reversed the induction observed in propionate-fed rats, but had no effect on standard-fed animals (Figure 1A).

We next investigated whether IGN induction was associated with an in vivo activation of cAMP-mediated signaling. After VIP injection, the intracellular cAMP content was increased 4-fold compared with saline-injected animals on standard diet. Similar cAMP levels were found in propionate-fed animals. Again, antagonism of VPAC1R abolished this increase (Figure 1B). The increase in cAMP is a key signal triggering the phosphorylation of cAMP responsive element binding protein (CREB). We determined the effect of the aforementioned conditions on the phosphorylation state of CREB. Both VIP injection and propionate feeding increased the levels of phosphorylated CREB, while not affecting the expression of CREB protein. As was the case before, treatment with PG 97-269 reversed the induction only in propionate-fed animals (Figure 1C). Furthermore, propionate feeding effectively increased the expression of phosphorylated CREB specifically in the enterocytes, as assessed by immunohistochemical methods (Figure S1). G6pc gene is endowed with CREB binding sites(10). To further document the regulation of this gene in the intestine, we studied the binding of phosphorylated CREB to the G6pc promoter by chromatin immunoprecipitation (ChIP). As shown in Figure 1D, the same pattern of activation/no activation was observed when studying the binding of phosphorylated CREB to the G6pc promoter.

Since VIP is expressed in enteric neurons, we examined if there was a variation of VIP expression in both enteric nervous plexuses. Using VIP immunofluorescence, we quantified VIP-positive cells in the myenteric and submucosal plexuses, in standard- and propionate-fed rats. In agreement with the observation that VIP is involved in propionate-mediated activation of IGN, propionate feeding effectively increased the ratio of VIP-positive neurons in the submucosal enteric plexus, which is primarily involved in the control of intestinal epithelial functions(6) (Figure 1E and G). No such increase was observed in the myenteric plexus (Figure 1F). However, caution is warranted since the absence of increase in VIP myenteric neurons could have been missed due to a technical issue. Indeed, VIP expression in the myenteric plexus is often studied following colchicine treatment to block its fast anterograde axonal transport, thereby retaining it in the cell body and facilitating labeling. This was not done here.

The purpose of this study was to investigate whether VIP was an enteric relay in the activation of gluconeogenic genes in the intestine in response to portal glucose signals relayed by the central nervous system (here activated by propionate). We show that VIP can induce 
G6Pase activity in vivo. Moreover, our data strongly suggest that VIP is necessary for propionate-mediated activation of IGN, since blocking of VPAC1 receptors in propionate-fed animals reverses the activation. These elements support the role of VIP as a local neuromodulator released by intrinsic neurons and responsible for the induction of IGN through a VPAC1-dependent mechanism in enterocytes.

VIP neurons are present in submucosal and myenteric plexuses. VIP neurons of the myenteric plexus receive strong vagal inputs(11) while neurons in the submucosal plexus directly regulate enterocyte functions(6). Given the fact that propionate feeding activates neurons of the dorsal motor nucleus of the vagus(5) and that the secretomotor activity of VIP has been linked to VPAC1 activation in either enterocytes or cholinergic enteric neurons of the submucosal plexus(12), it is very likely that activation of IGN may take place through the same pathway.

The involvement of VIP signaling in glucose and energy homeostasis is not restricted to the intestinal area. For example, VIP signaling is needed for normal glucose-mediated insulin release in the endocrine pancreas(13). Interestingly, VIP-null mice have elevated plasma glucose and enhanced taste preference for sweet(14), and streptozotocin-treated diabetic mice have decreased levels of gastrointestinal VIP(15). All these alterations point to a crucial role of gastrointestinal VIP signaling in maintaining whole body glucose homeostasis.

In conclusion, we report here a mechanism by which a nervous signal from the central nervous system activates intestinal glucose production, a function promoting numerous metabolic benefits(1-5). These findings provide a novel understanding of the brain-gut neural relationships. Given the beneficial outcomes deriving from the activation of IGN, this may open novel perspectives in the treatment and prevention of metabolic diseases.

\section{ACKNOWLEDGEMENTS}

All protocols were performed in accordance to our local ethics committee at Claude Bernard University Lyon 1.

The authors thank the CNRS (G.M.), the INRA (A.G.-S.), the Ministère de l'Enseignement Supérieur et de la Recherche (F.D.V.), and the Ecole Normale Supérieure de Lyon (F.P.) for funding their positions. This work was funded by the INSERM.

\section{AUTHOR CONTRIBUTIONS}


FDV and GM were responsible for the study concept; FDV, FP and AGS performed the experiments; FDV, FP, AGS and GM analyzed and interpreted the data; FDV, FP and GM wrote the manuscript.

\section{CONFLICTS OF INTEREST}

The authors disclose no conflicts of interest.

\section{REFERENCES}

1. Mithieux G, Andreelli F, Magnan C. Intestinal gluconeogenesis: key signal of central control of energy and glucose homeostasis. Curr Opin Clin Nutr Metab Care 2009;12:419423.

2. Mithieux G. Nutrient control of hunger by extrinsic gastrointestinal neurons. Trends Endocrinol Metab 2013;24:378-384.

3. Mithieux G, Misery P, Magnan C, Pillot B, Gautier-Stein A, Bernard C et al. Portal sensing of intestinal gluconeogenesis is a mechanistic link in the diminution of food intake induced by diet protein. Cell Metab 2005;2:321-329.

4. Duraffourd C, De Vadder F, Goncalves D, Delaere F, Penhoat A, Brusset B et al. Muopioid receptors and dietary protein stimulate a gut-brain neural circuitry limiting food intake. Cell 2012;150:377-388.

5. De Vadder F, Kovatcheva-Datchary P, Goncalves D, Vinera J, Zitoun C, Duchampt A, Bäckhed F, Mithieux G. Microbiota-Generated Metabolites Promote Metabolic Benefits via Gut-Brain Neural Circuits. Cell 2014;156:84-96.

6. Furness JB, Jones C, Nurgali K, Clerc N. Intrinsic primary afferent neurons and nerve circuits within the intestine. Prog Neurobiol 2004;72:143-164.

7. Zimmerman RP, Gates TS, Mantyh CR, Vigna SR, Welton ML, Passaro EP Jr, Mantyh PW. Vasoactive intestinal polypeptide receptor binding sites in the human gastrointestinal tract: localization by autoradiography. Neuroscience 1989;31:771-783.

8. Gautier-Stein A, Zitoun C, Lalli E, Mithieux G, Rajas F. Transcriptional regulation of the glucose-6-phosphatase gene by cAMP/vasoactive intestinal peptide in the intestine. Role of HNF4alpha, CREM, HNF1alpha, and C/EBPalpha. J Biol Chem 2006;281:31268-31278.

9. Prieto JC, Laburthe M, Rosselin G. Interaction of vasoactive intestinal peptide with isolated intestinal epithelial cells from rat. 1. Characterization, quantitative aspects and structural requirements of binding sites. Eur J Biochem 1979;96:229-237.

10. Gautier-Stein A, Mithieux G, Rajas F. A distal region involving hepatocyte nuclear factor 4alpha and CAAT/enhancer binding protein markedly potentiates the protein kinase A stimulation of the glucose-6-phosphatase promoter. Mol Endocrinol 2005;19:163-174. 
11. Berthoud HR. Morphological analysis of vagal input to gastrin releasing peptide and vasoactive intestinal peptide containing neurons in the rat glandular stomach. J Comp Neurol 1996;370:61-70.

12. Fung C, Unterweger P, Parry LJ, Bornstein JC, Foong JPP. VPAC1 receptors regulate intestinal secretion and muscle contractility by activating cholinergic neurons in guinea pig jejunum. Am J Physiol Gastrointest Liver Physiol 2014;306:G748-G758.

13. Winzell MS, Ahrén B. Role of VIP and PACAP in islet function. Peptides 2007;28:1805-1813.

14. Martin B, Shin Y-K, White CM, Ji S, Kim W, Carlson OD, Napora JK, Chadwick W et al. Vasoactive intestinal peptide-null mice demonstrate enhanced sweet taste preference, dysglycemia, and reduced taste bud leptin receptor expression. Diabetes 2010;59:1143-1152.

15. Adeghate E, Ponery AS, Sharma AK, El-Sharkawy T, Donáth T. Diabetes mellitus is associated with a decrease in vasoactive intestinal polypeptide content of gastrointestinal tract of rat. Arch Physiol Biochem 2001;109:246-251.

16. Croset M, Rajas F, Zitoun C, Hurot JM, Montano S, Mithieux G. Rat small intestine is an insulin-sensitive gluconeogenic organ. Diabetes 2001;50:740-746.

\section{ABBREVIATIONS}

ChIP: Chromatin immunoprecipitation; CREB: cAMP responsive element binding protein; FFAR3: Free fatty acid receptor 3; G6Pase: Glucose-6-phosphatase; G6PC: Glucose-6phosphatase catalytic subunit; IGN: Intestinal gluconeogenesis; PEPCK: Phosphoenolpyruate carboxykinase; VIP: Vasoactive Intestinal Peptide

\section{FIGURE LEGENDS}

Figure 1. VIP signaling mediates induction of intestinal gluconeogenesis. After two weeks on a standard or propionate-enriched diet, 3h-fasted rats were injected with VIP, VPAC1 antagonist PG 97-269 or only a saline solution.

A. Glucose-6-phosphatase activity in the jejunum of the rats. B. Intracellular cAMP in the jejunum of the rats. C. Effect of VIP signaling on CREB phosphorylation. Total CREB and pCREB proteins were assessed with western blots on intestinal lysates. D. ChIP assay of CREB occupancy over the rat $G 6 p c$ promoter.

Data are mean $\pm \mathrm{SEM}$ of $\mathrm{N}=6$ rats per group. $* * *, P<0.001$ vs. standard + saline, one-way ANOVA followed by Dunnett's post-hoc test. 
E to G. Increase in VIP-positive neurons in myenteric and submucosal plexuses by propionate feeding. E and F. Box plot showing 5 to 95 percentiles. Points below and above the whiskers are drawn as individual dots. VIP-positive cells were counted in 25-32 enteric plexuses of $\mathrm{N}=3$ rats per group. ${ }^{* *}, P<0.01$, Mann-Whitney's sum of rank test. G. Representative jejunal sections of VIP-positive and hematoxylin-stained cells of rats fed a standard or a propionate-enriched diet. Enteric plexuses are lined in white, VIP-positive and negative neurons are tagged respectively with short and full arrows. SMP: submucosal plexus; MEP: myenteric plexus; CML: circular muscle layer; LML: longitudinal muscle layer. Scale bar: $20 \mu \mathrm{m}$.

\section{SUPPORTING INFORMATION}

Figure S1. Representative sections of phosphorylated CREB-labeled intestinal cells (black arrows in the epithelium) of standard- and propionate-fed rats. Scale bar: $20 \mu \mathrm{m}$.

\section{MATERIALS AND METHODS}

\section{Animals and housing}

Adult male Sprague-Dawley rats (Charles River, France), aged 6 to 8 weeks and weighing 260-280 $\mathrm{g}$ at the time of their arrival, were housed in a climate-controlled room $\left(22 \pm 2^{\circ} \mathrm{C}\right)$ subjected to a 12-hour light/dark cycle with lights on at 7:00 AM, with free access to food and water. Standard diet was SAFE A04 (Augis, France). Sodium propionate (Sigma-Aldrich) was incorporated into the diet at $5 \%$ weight/weight. After two weeks of diet, animals were fasted for 3 hours and given an injection of either $0.9 \% \mathrm{NaCl}$ (saline), or $100 \mu \mathrm{g} / \mathrm{kg}$ body weight VIP (Bachem) or PG 97-269 (Bachem).

\section{Intestine sampling and G6Pase activity}

Rats were anesthetized 3 hours after drug injection with a single intraperitoneal injection of sodium pentobarbital $(100 \mathrm{mg} / \mathrm{kg})$ and the intestine was sampled as described previously(16). $10 \mu \mathrm{m}$-thick sections of the jejunum were used in histology experiments.

Frozen intestine samples were grinded to powder with a stainless steel mortar bathing in liquid nitrogen. The powder was further homogenized in $10 \mathrm{mM}$ HEPES and $0.25 \mathrm{M}$ sucrose, pH 7.4 (in a 9:1 volume ratio) and sonicated. G6Pase activity and non-specific phosphatase 
activity were assessed at maximum velocity in duplicates for each homogenate after addition of respectively $20 \mathrm{mM}$ glucose-6-phosphate or $\beta$-glycerophosphate (Sigma-Aldrich). After 10 minutes at $30^{\circ} \mathrm{C}$, the reaction was arrested by addition of excess $0.11 \mathrm{M}$ ascorbic acid in $10 \%$ trichloroacetic acid (Sigma-Aldrich). After centrifugation for 10 minutes at 2,000 g, the supernatant was collected. The released inorganic phosphate was assayed by spectrophotometry at $700 \mathrm{~nm}$ by complexation with molybdate ions (excess molybdate ions were precipitated by sodium meta-arsenite). Specific G6Pase activity was computed as the G6Pase activity minus the non-specific phosphatase activity in each sample. Protein concentrations were assayed by Bradford's method and activity was expressed in U/g of protein.

\section{Western Blot}

Tissue was grinded in $0.1 \mathrm{M} \mathrm{HCl}$ (CREB and pCREB). After denaturing electrophoresis, western blot was performed using rabbit anti-phospho CREB (1113-1, Epitomics, 1:500). For CREB blotting, pCREB membrane was stripped and western blot was performed using rabbit anti-CREB (1496-1, Epitomics, 1:1,000). Secondary antibody was goat anti-rabbit IgG, HRPconjugated (170-5046, Bio-Rad, 1:10,000).

\section{cAMP assay}

cAMP in the intestine was quantified using a direct cAMP enzyme immunoassay kit according to the manufacturer's instructions (ADI-900-066, Enzo Life Sciences).

\section{Chromatin immunoprecipitation (ChIP)}

ChIP was performed using Active Motif ChIP-IT® Express Enzymatic kit (53009), according to the manufacturer's instructions. Briefly, 50 to $100 \mathrm{mg}$ of frozen intestine was fixed in $1 \%$ formaldehyde in PBS-protein inhibitory cocktail. After chromatin harvesting and fragmentation, an input was prepared as a load control. After removing cross-links, DNA was purified by the phenol/chloroform method and quantified using a NanoDrop®. DNA integrity was verified by agarose gel electrophoresis. For immunoprecipitation, $10 \mu \mathrm{g}$ of chromatin were used with a rabbit anti-phospho CREB antibody (9191, Cell Signaling, 1:50). A GFP 
antibody (Abcam Ab6556) was used as a negative control. Q-PCR was performed using Faststart SYBR Green PCR reagents (Roche) with the following sequences on G6pc promoter:

- Forward GTTTGCTATTTTACGTAAATCACCCT

- Reverse CCTCTGCTATCAGTCTGTGCCTTGC.

\section{VIP immunofluorescence}

Jejunum sections were cleared of paraffin, rehydrated and incubated for 35 minutes at $95^{\circ} \mathrm{C}$ in citrate buffer for antigen retrieval. Sections were incubated in a blocking solution (PBS with $0.3 \%$ Triton X-100 and 5\% bovine serum albumin - 5\% goat serum) for 2 hours and further incubated with the primary antibody (rabbit anti-Neurofilament L, Millipore AB9568, 1:1,000) overnight at $4^{\circ} \mathrm{C}$. After several PBS rinses, sections were incubated with the secondary antibody (Alexa 633 goat anti-rabbit IgG, Molecular Probes A-21071, 1:2,000) for 2 hours at room temperature. After several rinses in PBS, a new step of blocking was performed for two hours. Sections were then incubated with rabbit anti-VIP antibody (Abcam Ab78536, 1:1,000) overnight at $4^{\circ} \mathrm{C}$, rinsed with PBS and incubated with Alexa 488 goat antirabbit IgG (Molecular Probes A-11008, 1:2,000) for two hours. Following rinses in PBS, sections were counterstained with $10 \mu \mathrm{g} / \mathrm{mL}$ Hoechst 33342 (Molecular Probes) and mounted using ProLong Gold ${ }^{\circledR}$ mounting medium (Molecular Probes). Specific staining was absent in the control experiments with omission of primary antibodies and no cross-reactivity was found between antibodies.

Images were obtained using a Carl Zeiss LSM 780 confocal microscope with 40x oilimmersion objectives. All photographs were imported to ImageJ (http://imagej.nih.gov/ij), adjusted for output and threshold to include all information-containing pixels, and adjusted for brightness and contrast to best demonstrate visible fluorescence labeling. No additional photographic alterations were performed. Colocalization of the two markers was quantified using Pearson's coefficient and scatter plot tools available with the software. Cells were counted in the submucosal and myenteric plexus and the proportion of VIP+ cells was obtained by examining co-expression with neurofilament $\mathrm{L}$.

\section{pCREB immunohistochemistry}


Jejunum sections were cleared of paraffin, rehydrated and incubated for 35 minutes at $95^{\circ} \mathrm{C}$ in citrate buffer for antigen retrieval. After 30 minutes of incubation with a peroxidase blocking solution (Dako kit K0690), sections were incubated in PBS with 0.3\% Triton X-100 with 5\% bovine serum albumin and 5\% goat serum for 2 hours and further incubated with the primary antibody (rabbit anti-pCREB, Epitomics 1113-1, 1:250) overnight at $4^{\circ} \mathrm{C}$. After several PBS rinses, sections were incubated with the secondary antibody (biotinylated goat anti-rabbit IgG, Vector BA-1000, 1:300) for 2 hours at room temperature. After several rinses in PBS, staining was performed using streptavidin-HRP and a chromogen reagent for 30 seconds (Dako kit K0690). Specific staining was absent in the control experiment with omission of primary antibodies.

Images were acquired using a light microscope (Nikon Eclipse E400) and Histolab software (Microvision Instruments).

\section{Statistical analysis}

GraphPad Prism ${ }^{\circledR}$ was used for statistical analysis. Groups were compared using one-way ANOVA followed by Dunnett's post-hoc test. The non-parametric Mann-Whitney's test was used for cell counting. $P<0.05$ was considered as statistically significant. 

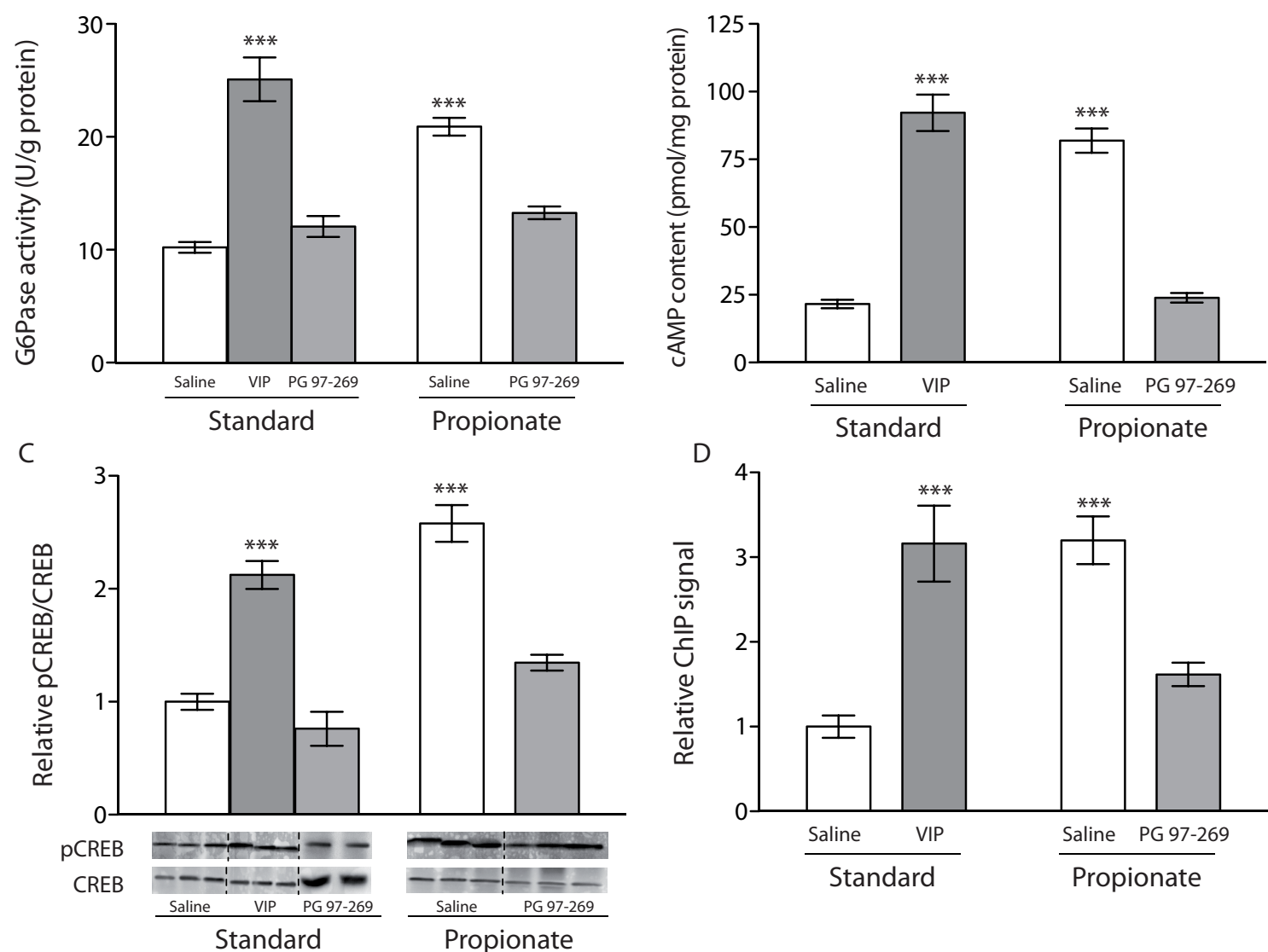

Submucosal plexus

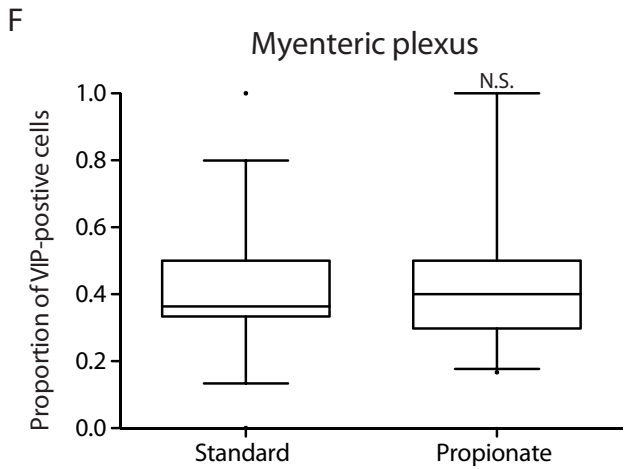

G

Standard

Propionate
Neurofilament

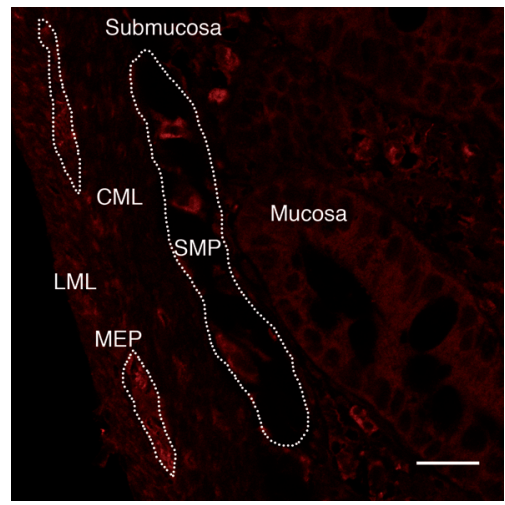

Submucosa

MEP
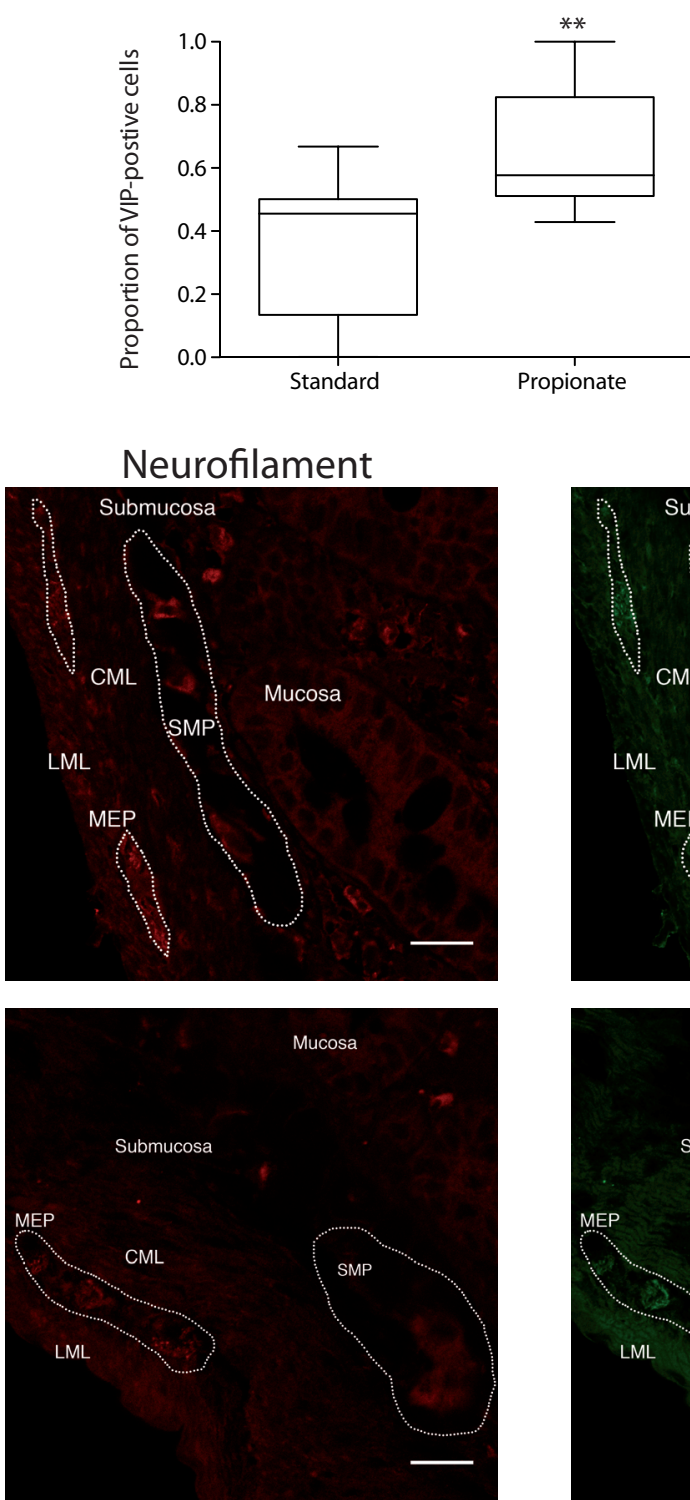

VIP

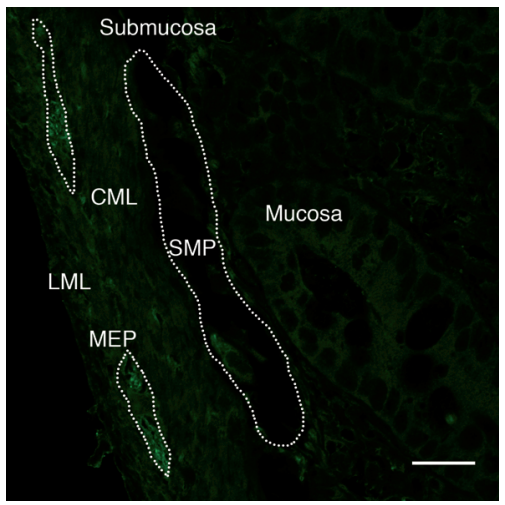

Mucosa

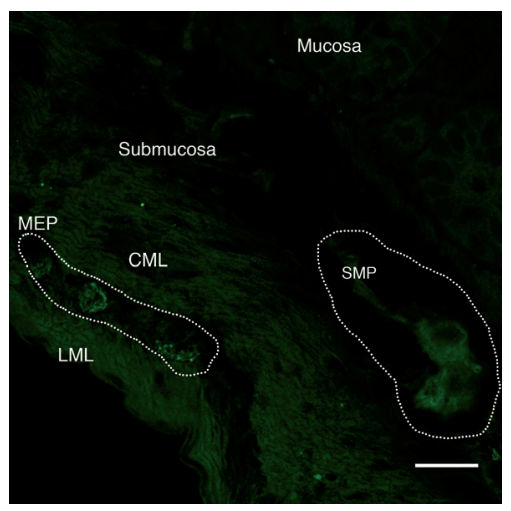

Merge

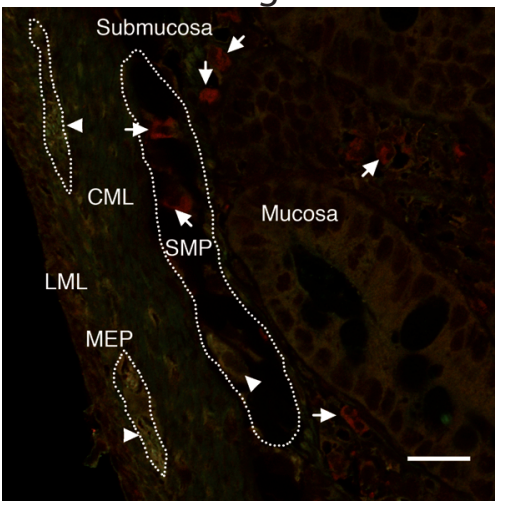

Mucosa

Submucosa

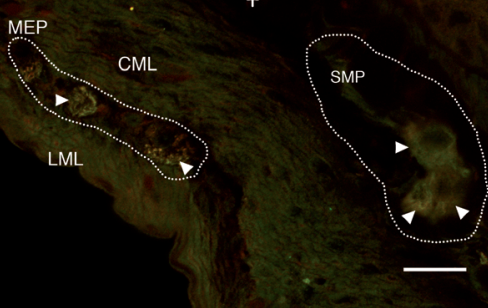

